\title{
Hautement différente : la génération $Y$, un défi de taille pour l'enseignement médical
}

\author{
Highly different: Generation Y, a major challenge for medical education
}

\author{
Francine BOULÉ \\ Département de médecine de famille, Faculté de médecine et des sciences de la santé 3001, 12e avenue Nord, \\ Sherbrooke (Québec), J1H 5N4, Canada
}

Manuscrit reçu le 6 juin 2011 ; commentaires éditoriaux formulés aux auteurs le 7 juillet 2011 et le 15 février 2012 ; accepté pour publication le 20 février 2012

Mots-clés
Éducation médicale ;
génération Y ; écarts
des générations ;
relations intergénéra-
tionnelles ; génération
du millénaire ;
apprenant
du millénaire

du millénaire

\section{Keywords}

Medical education; generation Y; generational gap; intergenerational relations;
Résumé - Introduction : La génération Y représente les jeunes nés entre 1980 et 1992, c'està-dire les actuels étudiants et résidents en médecine. Objectif : Cette synthèse de la littérature vise à identifier différentes particularités de la génération $\mathrm{Y}$, à documenter les défis pédagogiques que ces particularités constituent pour les professeurs et à élaborer des pistes de stratégies pédagogiques. Méthodes : La recherche d'articles de langue française et anglaise a été effectuée à partir des banques de données PubMed, CINAHL et ERIC, et manuellement dans les bibliographies des revues d'éducation médicale jusqu'en novembre 2010. Pour l'extraction des données, une démarche en trois étapes a été utilisée : 1) identification des problèmes-clés (particularités, défis, stratégies); 2) sélection des informations pertinentes dans les articles et 3) synthèse des données. Résultats : La stratégie de recherche a permis d'identifier 122 articles ; 68 ont été retenus pour évaluation. Les membres de la génération Y ont les caractéristiques suivantes : enfant-roi, surinvesti, issu de milieux structurés et confronté avec la peur ; adulte émergent, préoccupé par l'équilibre travail/vie personnelle, habité par les nouvelles technologies et l'activité multitâche ; étudiant avec des capacités d'apprentissage particulières, ennuyé par les cours magistraux, tracassé financièrement, confronté à une nouvelle image du médecin et recruté de façon différente. Conclusion : Un certain nombre de stratégies serait à préconiser dans l'enseignement aux apprenants issus de la génération $\mathrm{Y}$, parmi lesquelles : porter attention à la remise d'une évaluation difficile, favoriser la position d'apprentissage, expliciter les attentes, donner de la rétroaction régulière, faire participer les apprenants aux décisions, favoriser le travail collaboratif, modeler la réflexion critique et stimuler l'apprentissage au long de la vie, l'altruisme et les relations interpersonnelles, multiplier les options d'apprentissage et innover technologiquement.

Abstract - Introduction: Generation Y is made up of young people born between 1980 and 1992 who are currently medical students and residents. Objective: This literature review aims to: 1) identify the distinctive characteristics of Generation Y,2) document the challenges they represent for teachers, and 3) develop specific educational strategies. Methods: A research aimed at identifying publications in English or French was conducted in the PubMed, CINAHL and ERIC databases. It was completed by a detailed search through publication references. 
millennial generation; millennials; millennial learner
To retrieve the data, a three-phase process was used: 1) identifying key issues; 2) selecting relevant information and, 3) synthetizing the data. Results: The research strategy identified 122 articles. Sixty-eight articles were selected for analysis purposes. Generation Y includes pampered children whose parents overinvest in them. They grow up in structured homes and are overwhelmed by fear. As developing adults, they worry about balancing work and personal life, are surrounded by new technologies and are prone to multitasking. These students with unique learning abilities are easily bored by the traditional lecture format, worry about financial issues and are confronted today by the modern image physicians have. Various methods are used to recruit them. Conclusion: A number of learning strategies should be considered when teaching to Generation Y medical students, e.g. paying attention to the production of tough evaluations, promoting a learning attitude, detailing expectations, validating, fostering and providing regular feedback, involving learners in decision-making, using their skills, encouraging collaborative work, being available as a guide and a partner, shaping critical thinking, fostering a lifelong learning attitude, a passion to serve and interpersonal relationships, multiplying learning opportunities and innovating technologically.

\section{Introduction et problématique}

Les jeunes de la génération Y ou génération du millénaire sont nés entre 1980 et 1992. Enfants des babyboomers, ils représentent actuellement 70 millions d'Américains (États-Unis) (26\%) et 1,8 million de Québécois (20\%). Il s'agit de la deuxième génération en importance en nombre, après les boomers. C'est aussi la plus supervisée et la plus éduquée de l'histoire. Exposée à des expériences, des points de vue et des cultures extrêmement variés ${ }^{[1]}$ et avec la plus grande conscience sociale ${ }^{[2]}$, c'est la prochaine « grande » génération, celle qui forge la dynamique de la décennie actuelle. ${ }^{[3,4]}$ Hautement différente des générations précédentes, elle représente nos étudiants en médecine, nos résidents et nos jeunes professeurs ${ }^{[5]}$.

L'auteure est professeure au Département de médecine de famille de l'Université de Sherbrooke (Québec, Canada) depuis plus de 20 ans et joue le rôle de soutien aux étudiants en difficulté à titre d'adjointe à la vie étudiante depuis cinq ans. Préoccupée par certains enjeux qui ont changé depuis quelques années dans son travail quotidien auprès des étudiants en médecine et des résidents en médecine de famille, elle s'est demandé si des considérations générationnelles pourraient expliquer ces changements. Certaines autorités facultaires et départementales consultées partageaient le même questionnement. Ces enjeux ont été partagés au moyen d'un questionnaire soumis à 27 professeurs du Département de médecine de famille de l'Université de Sherbrooke, dont les résultats ont montré qu'ils ont aussi besoin de comprendre les enjeux de l'enseignement en lien avec les particularités de la génération $\mathrm{Y}$ dans une proportion de $92 \%$, leurs mécanismes d'adaptation aux défis de la vie adulte (77\%), leur façon d'acquérir les responsabilités professionnelles (96\%), de gérer leur stress (89\%), de concilier la vie personnelle et professionnelle $(93 \%)$ et de répondre à la surcharge de travail inhérente à la profession (100\%). Divers autres enjeux ont été mis en évidence : leur difficulté d'adaptation à un système en retard technologiquement, la familiarité entre le superviseur et le supervisé, leur difficulté à recevoir la rétroaction, les exigences des étudiants envers eux-mêmes et leur besoin d'avoir la meilleure évaluation.

Existe-t-il des facteurs générationnels chez les apprenants en médecine qui permettraient aux enseignants de mieux les connaître et les comprendre ? La littérature semble apporter divers arguments en ce sens et se révèle consensuelle dans plusieurs domaines en éducation, notamment quant au fait que les valeurs et les caractéristiques de tous les étudiants ne sont plus les mêmes ${ }^{[4]}$. Afin d'améliorer l'efficacité des professeurs et des professionnels impliqués dans les différentes étapes de la formation médicale, il faut commencer par mieux connaître les apprenants 
du millénaire et exploiter l'expérience de leur accompagnement tout au long de leur formation comme une opportunité de développement pédagogique ${ }^{[6]}$.

L'approche générationnelle considère les individus qui ont vécu des expériences communes comme ayant des attributs similaires ${ }^{[4,7]}$. Ils ont été élevés par des parents de la même génération et ils ont subi des influences socioculturelles et politico-économiques comparables. Ils ont écouté la même musique ${ }^{[4,5,8,9]}$. Comme chaque génération, ils ont une vision du monde différente de la génération qui les précède et de celle qui la suit, ainsi que des valeurs, des croyances et des attitudes qui leurs sont propres ${ }^{[10,11]}$. La prudence s'impose pour ne pas généraliser ; l'approche générationnelle ne suffit pas à elle seule à expliquer et à prédire tous les comportements des individus d'une génération donnée. Les différences peuventêtre attribuables à d'autres facteurs ${ }^{[12]}$, comme la situation dans le cycle de la vie, des effets de cohortes spécifiques ${ }^{[4]}$ ou des caractéristiques personnelles propres $^{[13-17]}$.

Ainsi, une meilleure compréhension de la génération Y pourrait permettre l'esquisse d'un guide utile pour l'enseignement ${ }^{[7,14]}$, de nature à optimiser les principes pédagogiques reconnus et à diminuer certaines incompréhensions entre les apprenants et les professeurs. La présente contribution se propose de rapporter une synthèse de la littérature visant à identifier différentes particularités de la génération $\mathrm{Y}$, à documenter les défis pédagogiques qu'elle représente pour les professeurs et à élaborer des pistes de stratégies pédagogiques.

\section{Méthodes}

Pour répondre à la question de recherche, l'auteure s'est d'abord appuyée sur une revue méthodique de la littérature relative aux sujets apprenants en médecine. Toutefois, le faible nombre d'articles sélectionnés a imposé d'étendre la recherche au domaine de la santé, en particulier aux apprenants en sciences infirmières, un domaine jugé connexe. La variété des types d'articles retenus (originaux ou d'opinions) et l'objectif de faire une recension exhaustive ont contribué à justifier le choix d'une synthèse de la littérature plutôt qu'une revue critique et systématique utilisant une grille d'analyse spécifique.

Inspirée d'un modèle transmissif d'enseignement, cette synthèse livre le contenu de la littérature recensée de façon à stimuler la réflexion des pédagogues mais ne prétend en aucune façon pouvoir en prescrire la conduite. Dans une perspective inductive, en cohérence avec la littérature recensée, la présentation des résultats s'articule autour du modèle conceptuel suivant : à partir de l'identification des particularités et des défis propres à une génération, des stratégies pédagogiques peuvent être déduites. La littérature a donc été révisée à la recherche de ces trois éléments et les résultats sont présentés ici selon ce modèle.

La revue de littérature a été effectuée à partir des banques de données accessibles grâce à l'interface PubMed (1950-novembre 2010), à la base de données Cumulative Index to Nursing and Allied Health Literature - CINAHL - (1937 à novembre 2010) et à la base de données Education Resources Information Center - ERIC - (1966-novembre 2010).

La stratégie de recherche pour des articles en français ou en anglais a utilisé les mots-clés et les termes MeSH suivants : millenial generation ou millennials ou generation $Y$ ou millennial learner ou generationnal gap ou education, medical et intergenerational relations; elle a permis de relever 122 articles. Cette stratégie a permis d'identifier seulement des articles d'auteurs anglophones, en majorité en provenance d'Amérique du Nord, mais aussi d'Australie et de la Nouvelle-Zélande.

À partir de la lecture des titres, des résumés ou des textes complets, 54 articles ont été éliminés, soit parce qu'ils ne concernaient pas du tout le sujet, qu'ils n'étaient pas relatifs aux étudiants du domaine de la santé, qu'ils ne concernaient pas la bonne génération ou qu'ils concernaient la génération en début de pratique médicale et non des apprenants en médecine.

Pour l'extraction des données, une démarche en trois étapes a été utilisée: 1) identification des problèmes-clés (particularités, défis, stratégies) ; 2) sélection des informations pertinentes dans les articles et 3 ) synthèse des données. 


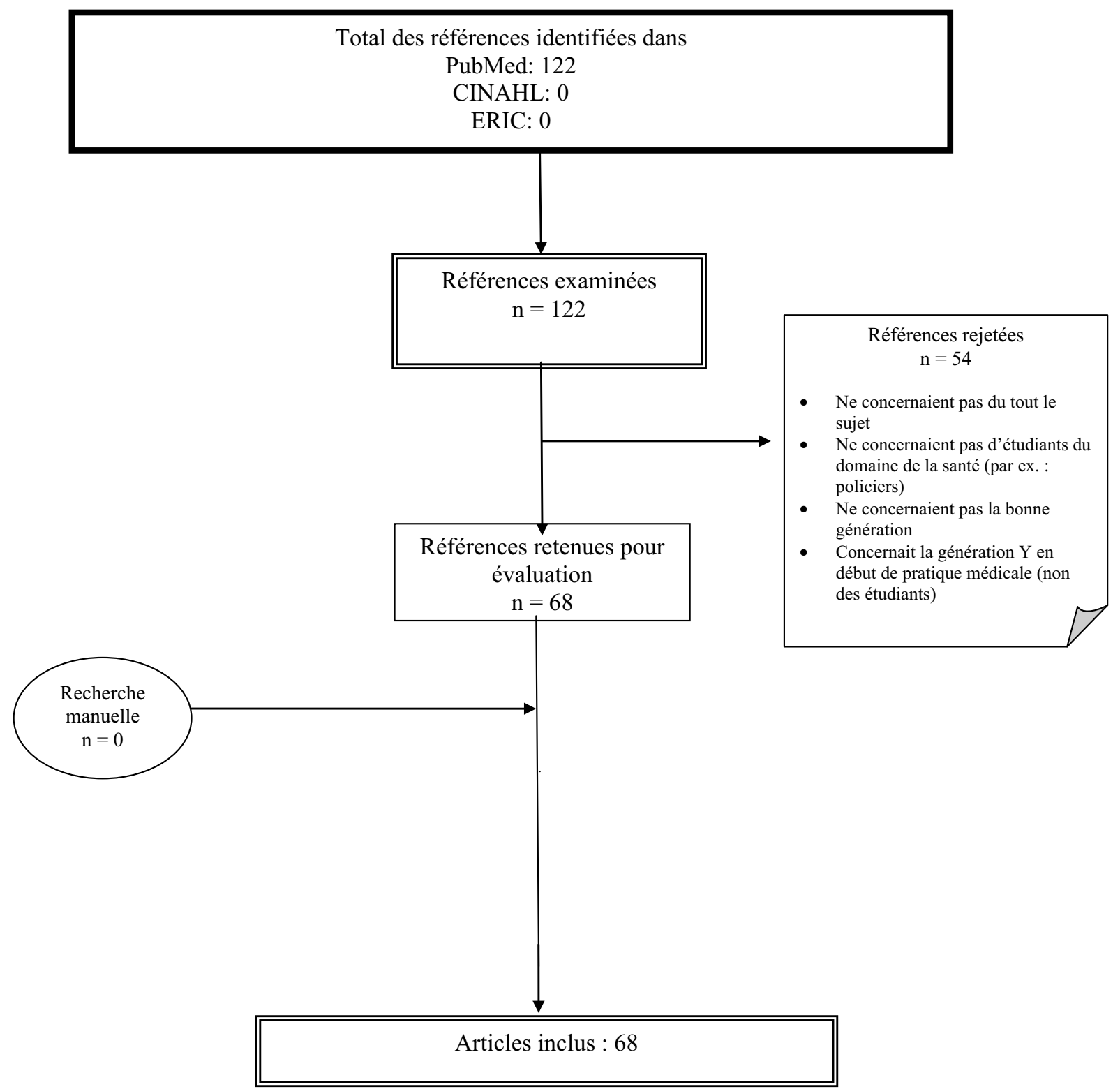

Fig. 1. Nombre de références identifiées dans les étapes de la synthèse.

\section{Résultats}

Au total, 68 articles ont été retenus, 31 concernant spécifiquement le domaine des études médicales ${ }^{[3-}$ $7,12,19-43]$. Vingt et un étaient en lien avec les études en sciences infirmières ${ }^{[8-10,13,15-16,44-58]}$, neuf avec différents domaines en santé ${ }^{[11,17,18,59-64]}$ et sept avec des domaines généraux pertinents comme le monde des affaires ${ }^{[1,2,14,65-68]}$. La figure 1 montre le nombre de références trouvées à chaque étape du processus de sélection. Tous les types d'articles ont été étudiés. La recherche d'articles complétée manuellement dans certaines revues pertinentes n'a pas permis de trouver d'article additionnel.

Les résultats issus de la littérature sont présentés comme suit: les particularités identifiées sont immédiatement suivies des défis qu'elles génèrent ainsi que des stratégies pédagogiques proposées. Les 
différentes particularités sont ordonnées à partir de la naissance des jeunes de la génération $Y$ jusqu'à leur recrutement en résidence, sous les rubriques : grandir, évoluer, apprendre.

\section{Génération Y : grandir}

\author{
L'enfant-roi devenu adulte
}

\section{Particularités}

Les enfants-rois ont le plus petit ratio enfants/parents de l'histoire ${ }^{[5]}$. Très désirés par leurs parents ${ }^{[39]}$, ils ont été élevés au moyen d'approches favorisant le développement de l'estime de soi à tout prix. Ils ont reçu un amour inconditionnel et des éloges constants, parfois démesurés, allant parfois jusqu'à l'abstention de critiques, même devant des comportements inappropriés ${ }^{[5]}$.

Ils ont été impliqués dans de nombreuses activités parascolaires, où il n'y a eu que des gagnants ( « trophy generation ») ${ }^{[3]}$. Ils ont été protégés des sentiments d'échecs. Ils ne se sont pas fait dire non souvent. Des études ont démontré qu'ils ont plus de caractéristiques narcissiques et sont plus perfectionnistes que les générations précédentes ${ }^{[6]}$.

\section{Défis}

Devenus adultes, lorsque les Y affrontent une difficulté, leur détresse peut être grande car ils ont été peu confrontés à l'échec. Toute critique à leur endroit tend à diminuer leur estime de soi ${ }^{[39]}$. Leur réaction à la critique n'attire pas toujours la sympathie de leurs collègues des générations antérieures ${ }^{[69]}$, d'autant que le perfectionnisme, que l'on retrouve particulièrement chez les étudiants en médecine, les fragilise davantage face aux évaluations difficiles ${ }^{[6]}$.

\section{Stratégies pédagogiques}

La présentation d'une évaluation doit être particulièrement objective et constructive si elle comporte des points à améliorer. Pour être en mesure de corriger un problème, les apprenants de cette génération ont besoin de bien comprendre la raison qui impose le changement. Ils ont besoin de participer à la prescription pédagogique pour rester engagés dans la gestion de leur apprentissage ${ }^{[13]}$. Ils doivent apprendre à reconnaître leurs erreurs et apprendre à partir de celles-ci. Le professeur doit être un modèle pour eux en ce sens, en reconnaissant ses propres erreurs avec humilité ${ }^{[5]}$

\section{Victimes de surinvestissement}

\section{Particularités}

Les membres de la génération $Y$ ont été victimes de surinvestissement de la part de parents qui, travaillant tous les deux, se sont sentis coupables d'être moins présents. Ils les ont donc surprotégés pour compenser. Les jeunes ont été à tout moment supervisés, «survolés » par leurs parents très impliqués, dits « parents-hélicoptères », prêts à intervenir à chaque instant dans tous les aspects de leur vie.

Ils ont énormément échangé avec leurs parents sur une grande variété de sujets ${ }^{[3]}$. Ils ont eu le droit de poser toutes les questions qui leur venaient à l'esprit. Ils ont été consultés sur tout. Ils ont appris à se considérer comme des partenaires égaux à la fois de leurs parents et de leurs professeurs.

\section{Défis}

Comme ils n'ont jamais vraiment compté sur euxmêmes, ils n'ont pas développé une grande confiance en eux et ils demeurent dépendants plus longtemps. Ils ont des difficultés à prendre des décisions par euxmêmes et à gérer leurs propres agendas, notamment $^{[6]}$. Ils ont appris à respecter les règles mais, assoiffés de guidage, ils sont exigeants, ont beaucoup $\mathrm{d}$ 'attentes et posent beaucoup de questions ${ }^{[3]}$.

La reconnaissance représente un motivateur-clé pour eux ${ }^{[8]}$. L'absence de rétroaction positive représente en soi l'équivalent d'une rétroaction négative ${ }^{[68]}$. Ils apprécient le contact personnel direct avec leurs professeurs ${ }^{[18]}$ et ils veulent que l'on tienne compte de leur opinion ${ }^{[1,49]}$. Ils ne veulent pas être des spectateurs passifs ${ }^{[69]}$. 


\section{Stratégies pédagogiques}

Les apprenants de la génération Y sont à l'aise dans un milieu structuré et interactif où l'ambiguïté est minime ${ }^{[6]}$, où ce qu'il faut apprendre est explicite $^{[4,5,59,18]}$. Il faut offrir un milieu où peuvent être satisfaits leurs grands besoins de validation, d'approbation et d'encouragement ${ }^{[47,51,68]}$, grâce à une rétroaction fréquente, courte et immédiate sur leur performance ${ }^{[2,3,5,8,14]}$ et où ils participent aux décisions ${ }^{[13]}$. Les professeurs doivent être disponibles rapidement, être proches des apprenants et s'attendre à répondre à beaucoup de questions ${ }^{[3,14]}$.

\section{Activités d'équipe programmées à la minute}

\section{Particularités}

Leur vie a toujours été très structurée et remplie d'activités programmées dès la naissance. Ils ont été précocement habitués à travailler en équipe, à la crèche comme à l'école. De plus, comme leurs parents leur ont répété qu'ils sont « uniques » et qu'ils peuvent faire « la différence », ils y croient et perçoivent une injonction d'excellence ${ }^{[3,39]}$.

\section{Défis}

Ils veulent changer le monde et croient en avoir le pouvoir. Ils pensent faire mieux et pouvoir éditer de nouvelles règles ${ }^{[3]}$. Comme ils sentent constamment une pression, ils ne veulent pas perdre de temps dans des activités d'apprentissage qu'ils jugent inutiles ou qu'ils ne peuvent appliquer immédiatement ${ }^{[3]}$. Leur façon de contester la pertinence des apprentissages peut les faire paraître arrogants ${ }^{[14]}$. Chez certains, cette caractéristique peut donner tendance à être confiants indûment au point de ne pas reconnaître qu'ils ont besoin de s'améliorer («overconfident $)^{[6,18]}$.

\section{Stratégies pédagogiques}

Le travail collaboratif dans lequel ils excellent est primordial et peut même les réconforter ${ }^{[7,9,18]}$. Comme ils ne veulent pas perdre de temps, il faut revoir la qualité des contenus et la quantité des cours, trouver des alternatives d'apprentissage au moyen de nouvelles technologies et les planifier à un moment qui n'interfère pas avec le temps réservé aux activités personnelles ou familiales ${ }^{[42]}$.

La peur au rendez-vous

\section{Particularités}

Les évènements qui sont survenus au début de leur vie (10-25 ans) ont une influence profonde et durable sur les attitudes et les préférences des individus ${ }^{[12,13]}$. La génération $\mathrm{Y}$ a été affectée par plusieurs évènements significatifs, le terrorisme étant au premier plan avec les attentats terroristes aux États-Unis, ceux d'Oklahoma City en 1995 et ceux du 11 septembre 2001 à New York et avec la guerre antiterroriste qui a suivi en Iraq et en Afghanistan. De plus, la tuerie de masse à Columbine High School les a marqués. Le réchauffement de la planète, la crise de l'énergie, la crise économique internationale et la mondialisation les influencent également ${ }^{[3]}$.

\section{Défis}

Ils vivent dans la peur (la vie peut s'arrêter à tout moment $)^{[3]}$, ce qui leur procure un profond besoin de servir leur communauté et génère un grand besoin d'affiliation ${ }^{[19]}$ et ce, parfois aux dépens de la capacité de leadership et du désir d'influencer les autres, qui étaient des caractéristiques propres aux générations précédentes ${ }^{[27]}$.

\section{Stratégies pédagogiques}

Il faut leur donner l'opportunité de s'associer à une cause, de s'investir dans un projet porteur de sens et de développer et maintenir des relations authentiques, de créer un esprit d'équipe et d'appartenir à un groupe social. Il faut tenir compte que le soutien des amis et des parents est aussi très important ${ }^{[3]}$. Une formation pour favoriser le développement d'habiletés de leadership est pertinente et nécessaire ${ }^{[19]}$. 


\section{Génération $Y$ : évoluer}

\author{
Ni adolescent, ni adulte
}

\section{Particularités}

Arnett $^{[50]}$ propose d'individualiser un stade de développement personnel, entre 18 et 24 ans, qui ne représente ni la fin de l'adolescence ni le début de l'âge adulte (stades de développement proposés par Erikson). Une dépendance prolongée des parents ainsi qu'un retard dans l'engagement avec une autre personne ${ }^{[50]}$ signalent une nouvelle transition développementale nommée «l'adulte émergent». Cette phase est caractérisée par une exploration intense de l'identité, de plus en plus d'introspection et des changements personnels fréquents, qui expliquent entre autres la difficulté des jeunes à effectuer un choix de carrière. Ils explorent plusieurs relations interpersonnelles différentes et confrontent les valeurs familiales.

\section{Défis}

Durant cette période, l'individu doit acquérir l'autosuffisance, prendre des décisions de façon autonome, agir de façon responsable, être indépendant financièrement.

\section{Stratégies pédagogiques}

Ils répondent habituellement favorablement à des conseils sur le développement d'habiletés organisationnelles, sur des stratégies de gestion du temps, sur la prise de décision autonome et sur le sens des responsabilités. Il faut savoir qu'il s'agit d'un processus dynamique qui s'étale sur plusieurs années ${ }^{[50]}$.

\section{Équilibre travail-vie personnelle}

\section{Particularités}

La génération $Y$ valorise le temps pour soi et pour la famille plus que toutes les autres générations ${ }^{[16,35]}$.
Former un couple et avoir des enfants est bien plus important que la carrière ${ }^{[19]}$. Avoir du temps est plus important qu' avoir de l'argent ${ }^{[12]}$. Plusieurs étudiants ne sont pas prêts à s'investir dans l'excellence professionnelle si c'est aux dépens de leur santé personnelle ou de la qualité du temps passé en famille. Ils croient qu'une vie bien équilibrée en dehors de la médecine fait d'eux de meilleurs médecins ${ }^{[5,6,12,41]}$. Contrairement à ce qu'on aurait été tenté de croire, cet état n'est pas en lien avec la féminisation de la médecine ${ }^{[30,43]}$.

\section{Défis}

Il faut faire face au paradoxe de l'équilibre personnel qui se heurte à la demande croissante de la communauté qui est à la fois plus exigeante et vieillissante ${ }^{[23]}$.

\section{Stratégies pédagogiques}

Il faut tenter de leur montrer que le fait de travailler davantage occasionnellement ne nuit pas nécessairement à leur qualité de vie et qu'ils ne pourront qu'être fiers d'eux ${ }^{[21]}$. De plus, les professeurs doivent être réalistes avec les étudiants au sujet des défis que posent la résidence (internat) et la pratique professionnelle; ils doivent leur expliquer que, du moins pour un temps, leur travail exercera des contraintes sur le reste de leur vie et il conviendra de les soutenir dans ces circonstances ${ }^{[6]}$. Comme les études ont démontré que la seule différence dans le professionnalisme chez la génération $Y$ est l'altruisme et qu'il se construit plus au fil de la formation et de la pratique ${ }^{[30]}$, les enseignants peuvent se permettre d'être patients avec eux en les guidant doucement vers la passion de servir les autres $^{[61]}$.

Nés avec une souris à la main

\section{Particularités}

Les apprenants de la génération $\mathrm{Y}$ appartiennent à la «Net Generation ». Ils n' ont jamais connu le monde sans Internet, ce qui est extrêmement marquant pour 
eux. L'utilisation des technologies informatiques fait partie intégrante de leur vie. Ils semblent l'utiliser comme extension de leur cerveau ${ }^{[67]}$. Ils manipulent l'information différemment. Ils ont un accès instantané et continu à de l'information sur une grande variété de sujets. Ils utilisent «Google» pour leurs recherches. Ils partagent l'information efficacement sur Internet, en direct ${ }^{[4]}$.

Ils n'ont pas envie d'apprendre par cœur ce qui est facilement disponible sur Internet et qui sera probablement dépassé quand ils commenceront à travailler $^{[14]}$. Ils ont aussi beaucoup joué à des jeux vidéo dès leur jeune âge, ce qui a cultivé une méthode d'apprentissage par essais et erreurs ${ }^{[4,10]}$.

\section{Défis}

Ils s'ennuient facilement ${ }^{[13]}$. Ils ont une «tolérance zéro » aux retards, à l'ère des guichets automatiques bancaires et de la banque et des achats en ligne ${ }^{[54]}$. Ils attendent donc de leurs supérieurs une immédiateté dans la réponse à leurs questions qui peuvent maintenant être posées par courriel et même par messagerie texte. Ils sont ouverts pour partager des détails personnels sur Internet, acceptant le risque d'accès par des étrangers, parmi lesquels des patients parfois avides de renseignements personnels sur leur médecin ${ }^{[4,38]}$.

\section{Stratégies pédagogiques}

Il faut parler ouvertement de l'éventualité qu'ils puissent devoir attendre pour avoir des réponses ou des solutions ${ }^{[4]}$ dans le cadre de leurs situations d' apprentissage, particulièrement en pratique médicale, en soulignant qu'ils n'auront pas toujours toutes les réponses aussi rapidement qu'ils ne le voudraient et qu'ils devront faire face à des situations sans solution $^{[46]}$. Il faut repenser les perspectives d'enseignement en tenant compte de certains éléments : l'information théorique est plus facile à trouver, le rôle de professeur est plus que jamais un rôle de guide, de mentor, de partenaire ${ }^{[54]}$. Le professeur doit modéliser la contemplation tranquille, la réflexion critique et la curiosité. Il doit sensibiliser les étudiants à la réalité de soigner des patients « branchés » $\mathrm{e}^{4,38,54]}$. De plus, il faut leur faire prendre conscience du fait que les nouvelles technologies ne remplacent pas une interaction en face à face avec les collègues et les superviseurs et ce, dans plusieurs circonstances ${ }^{[18]}$, en modélisant explicitement le dialogue avec les pairs ${ }^{[49]}$. La stratégie consistant à les faire parler d'eux-mêmes est un bon moyen d'augmenter leurs habiletés de relations interpersonnelles dont la technologie les a privés ${ }^{[13]}$.

\section{Efficaces en activité multitâche?}

\section{Particularités}

La grande accessibilité et l'omniprésence des nouvelles technologies ont habitué les apprenants de la génération $\mathrm{Y}$ à effectuer plusieurs tâches à la fois et ils en sont fiers. L'avantage des dispositifs permettant l'activité multitâche est un accès facile et varié à différentes sources d'information ${ }^{[14]}$. Cependant, le traitement de plusieurs sources d'information en même temps pose un défi au niveau cognitif, en affectant la cible de l'apprentissage et la concentration. La recherche en psychologie cognitive démontre qu'on ne peut pas vraiment transférer efficacement notre attention d'une tâche à l'autre sans nuire à la performance ${ }^{[6]}$. Ainsi, lorsqu'ils font plusieurs tâches à la fois, il leur est plus difficile de se rappeler des informations car l'encodage en mémoire moins performant nuit à la récupération des données ${ }^{[4]}$. Ils sont facilement déconcentrés ${ }^{[5]}$.

\section{Défis}

L'activité multitâche n' est pas efficace pour les tâches intellectuelles. Les ressources cognitives sont moindres. Les apprenants qui s'y livrent retiennent moins l'information et il leur est plus difficile de sélectionner les données à partir de leur mémoire pour effectuer une tâche complexe.

\section{Stratégies pédagogiques}

Il faut informer explicitement les étudiants des résultats de la recherche en psychologie cognitive, qui montrent la vulnérabilité cognitive qu'induit la ten- 
tative d'effectuer plusieurs tâches en même temps et tenter de les décourager d'adopter un fonctionnement multitâche.

\section{Génération $Y$ : apprendre}

\section{Une capacité d'apprentissage différente}

\section{Particularités}

Les étudiants de la génération $\mathrm{Y}$ ont une capacité d'apprentissage nettement différente de celle des générations précédentes. La jeune génération réussit mieux les tests d'intelligence (quotients intellectuels plus élevés $)^{[19]}$ mais leurs habitudes de lecture ont diminué de façon importante ; en effet, moins de $50 \%$ aiment lire ${ }^{[6,50]}$. Moins de la moitié des jeunes ont une bonne compréhension des concepts de base en mathématiques, lecture et écriture ${ }^{[67]}$. Ils ont donc souvent plus de difficulté à communiquer de façon traditionnelle (par écrit). Le tiers de la génération Y étudie de façon irrégulière et non planifiée et, lorsqu'ils s'y mettent, ils s'ennuient rapidement et délaissent leurs études au profit d'une autre activité après peu de temps ${ }^{[4]}$. Ils manquent de pensée indépendante et critique et démontrent un manque de flexibilité pour composer avec des situations ambiguës ${ }^{[4,14,39]}$.

Il semble y avoir un écart entre le désir de réussir, qui est grand parmi ces jeunes, et les efforts à fournir $^{[4]}$. Les étudiants du millénaire ont de meilleurs résultats d'évaluation pour une quantité moindre de travail fourni ${ }^{[6]}$. Ils sont davantage concernés par les notes obtenues, en tant que mesure de leur apprentissage, que par l'utilité de cet apprentissage ${ }^{[14,54,55]}$.

Au début de la formation universitaire, il y a une transition scolaire qui représente un défi pour les apprenants. Ils ne reçoivent plus l'information de façon passive et ils réalisent qu'il existe de l'incertitude et de l'ambiguïté dans l'apprentissage et des points de vue différents. Ils doivent devenir participants actifs de leur formation ${ }^{[50]}$.

\section{Défis}

Enseigner à des étudiants en médecine, qui montrent un certain désengagement pédagogique car ils n'ont pas toujours la capacité de fournir les efforts nécessaires à la réussite tant en quantité qu'en qualité ${ }^{[4]}$, se heurtant à plusieurs difficultés. Ils veulent de bonnes notes pour avoir « essayé » ou « travaillé fort », même si la performance est sous-optimale et que les objectifs ne sont pas tous atteints ${ }^{[6,14,61]}$. Leur expérience éducationnelle antérieure ne les a pas préparés aux défis et au travail nécessaire à la réussite. Ils doivent développer leurs compétences en utilisant des domaines qu'ils n'ont pas tout à fait maîtrisés préalablement ${ }^{[6]}$.

\section{Stratégies pédagogiques}

Ces étudiants sont ouverts au soutien pédagogique et ils bénéficient de plans d'apprentissage individualisés et variés ${ }^{[4]}$. Ils ont besoin de plusieurs options pour évoluer : ils apprennent beaucoup à partir de simulations et d'exemples cliniques, qui doivent apparaître tôt dans la formation ${ }^{[14]}$. Il faut que l'enseignement soit interactif, agréable et stimulant ${ }^{[39]}$. Il faut contribuer à créer un environnement où ils sont enthousiastes et curieux face à leur rôle d'apprenants et engagés à améliorer les faiblesses identifiées ${ }^{[50]}$.

Cours magistraux : désuets

\section{Particularités}

Comme de nombreux auteurs le confirment, la méthode d'enseignement qui consiste en des cours magistraux classiques est devenue nettement obsolète et inefficace ${ }^{[4]}$.

\section{Défis}

Il convient de les garder intéressés et stimuler leur attention ${ }^{[13]}$ car, en fait, ils ne veulent pas cesser complètement d'avoir des cours magistraux ${ }^{[55,59]}$. Ils préfèrent l'action à l'observation, ils sont plus visuels que verbaux ${ }^{[57]}$. 


\section{Stratégies pédagogiques}

Il faut utiliser davantage de ressources technologiques avancées, exploitant Internet, tels qu'un blogue ou un site de chat pour soutenir des apprentissages qu'ils peuvent poursuivre plus tard de façon autonome. Il faut varier les méthodes d'apprentissage pour combattre le manque d'attention. Des cas cliniques en petits groupes doivent être privilégiés ${ }^{[4-6]}$. Ces étudiants apprécient la nature interactive des simulations cliniques et le partage du vécu professionnel de leurs professeurs $^{[55]}$.

\section{Les dettes}

\section{Particularités}

Le coût élevé des études médicales représente une source de stress particulièrement importante pour les jeunes de la génération Y. Comme ils n'ont été privés de rien et qu'ils sont de grands consommateurs, ils empruntent de l'argent au-delà de leur capacité pour se procurer leurs biens.

\section{Défis}

L'accumulation de davantage de dettes d'étude par les étudiants de la génération Y soulève la question des conséquences que cet état de fait peut avoir sur leur statut économique et sur l'influence qu'il peut avoir sur leur choix de carrière ${ }^{[7]}$.

\section{Stratégies pédagogiques}

Envisager un système de bourses d'excellence et fournir aux étudiants concernés une formation sur la gestion et la planification financière sont des stratégies à explorer. Ces étudiants ont besoin de soutien, particulièrement au sujet de leur choix de carrière.

Image sociétale réaliste

\section{Particularités}

L'image traditionnelle du médecin a considérablement changé au cours des 20 dernières années. Le respect de la société pour le médecin et le prestige associé à la profession sont moins importants. L'engagement traditionnel et le don de soi de la part des médecins envers leur profession diminuent aussi. La médecine devient un travail comme un autre et n'est plus le centre de leur vie ${ }^{[5,19]}$. Les jeunes de la génération $\mathrm{Y}$ ont de multiples champs d'intérêts dans toutes sortes de domaines ${ }^{[3]}$.

\section{Défis}

Ces étudiants peuvent sembler moins intéressés et moins impliqués dans leur travail et leur motivation à donner des soins de qualité que le public attend peut paraître moindre ${ }^{[61]}$.

\section{Stratégies pédagogiques}

Il est nécessaire de développer les exigences du professionnalisme, en présentant les valeurs de base d'une bonne pratique médicale, parmi lesquelles la loyauté envers le patient. Les étudiants doivent bien comprendre ce qu'ils représentent pour les patients $^{[35,36]}$.

\section{Stratégies de recrutement}

\section{Particularités}

Pour qu'un programme de formation médicale demeure compétitif, il est primordial de s'adapter à la «Net Generation », de réinventer les stratégies de recrutement.

\section{Défis}

Le monde médical a beaucoup évolué technologiquement pour les applications cliniques, mais il a des difficultés à suivre le rythme en ce qui concerne le champ de l'éducation médicale ${ }^{[4]}$. Parallèlement, les méthodes de recrutement dans les programmes montrent aussi un retard.

\section{Stratégies pédagogiques}

Il faut être plus « technologique » pour attirer les nouveaux étudiants dans nos programmes. Puisqu'ils sont 
Tableau I. Particularités des étudiants de la génération « $Y$ » et stratégies pédagogiques envisageables à leur égard.

\begin{tabular}{|c|c|}
\hline Particularités des «Y» & Stratégies pédagogiques \\
\hline $\begin{array}{l}\text { Enfants-rois : peu confrontés à l'échec, } \\
\text { perfectionnistes }\end{array}$ & $\begin{array}{l}\text { Soigner la présentation d'une évaluation négative, favoriser la } \\
\text { position d'apprentissage }\end{array}$ \\
\hline Peu confiants & $\begin{array}{l}\text { Expliciter les attentes pour toute situation d'apprentissage, éviter } \\
\text { l'ambiguîté }\end{array}$ \\
\hline $\begin{array}{l}\text { Victimes de surinvestissement : surprotégés, } \\
\text { dépendants }\end{array}$ & $\begin{array}{l}\text { Valider, approuver, encourager, soutenir, donner de la rétroaction, } \\
\text { fréquente et immédiate. La reconnaissance est un motivateur clé. }\end{array}$ \\
\hline Ont été consultés sur tout & $\begin{array}{l}\text { Être disponible, répondre à leurs questions, les inciter à participer à } \\
\text { la prise des décisions, respecter leur initiative et leur compétence. }\end{array}$ \\
\hline $\begin{array}{l}\text { Programmés : experts du réseautage, } \\
\text { pression pour exceller }\end{array}$ & $\begin{array}{l}\text { Favoriser le travail collaboratif, interactif, stimulant, sans perte de } \\
\text { temps. }\end{array}$ \\
\hline $\begin{array}{l}\text { Peur : évènements sociopolitiques difficiles, } \\
\text { besoin d'affiliation }\end{array}$ & $\begin{array}{l}\text { Les encourager à s'associer à une cause, à s'investir dans un projet } \\
\text { porteur de sens. }\end{array}$ \\
\hline $\mathrm{Ni}$ ado ni adulte : adultes émergents & Les amener vers l'autosuffisance par des conseils spécifiques. \\
\hline Équilibre travail/vie personnelle important & $\begin{array}{l}\text { Les guider vers la passion de servir les autres (altruisme) tout en } \\
\text { respectant leur vie personnelle }\end{array}$ \\
\hline $\begin{array}{l}\text { Nés une souris à la main : habités par } \\
\text { internet }\end{array}$ & $\begin{array}{l}\text { Utiliser les nouvelles technologies tout en les invitant à la rigueur } \\
\text { dans le choix de l'information. Inspirer, modéliser la contemplation } \\
\text { tranquille, la réflexion critique, la curiosité, le dialogue avec les } \\
\text { pairs, l'excitation d'apprendre tout au long de la vie. }\end{array}$ \\
\hline Adeptes du multitâche & Remettre en cause les activités multitâche et en définir les règles. \\
\hline $\begin{array}{l}\text { Capacité d'apprentissage différente : plus } \\
\text { difficile }\end{array}$ & $\begin{array}{l}\text { Offrir un soutien pédagogique précoce et des méthodes } \\
\text { d'apprentissage variées et mises en contexte. }\end{array}$ \\
\hline Cours magistraux désuets : s'y ennuient & Innover dans les présentations magistrales. \\
\hline $\begin{array}{l}\text { Image sociétale réaliste : confrontés à une } \\
\text { image du médecin moins valorisée }\end{array}$ & $\begin{array}{l}\text { Leur inculquer les valeurs de base d'une bonne pratique médicale, } \\
\text { dont la loyauté envers le patient. }\end{array}$ \\
\hline Accumulent beaucoup de dettes & Les soutenir et les outiller dans la gestion des finances. \\
\hline Recrutés différemment & Optimiser et innover le recrutement \\
\hline
\end{tabular}

nés avec Internet, il faudrait leur permettre de l'utiliser dans les hôpitaux et même développer la communication par réseaux sociaux ${ }^{[3,60]}$. Les activités qui servent à la promotion des programmes doivent être très innovantes. Toutes les informations pertinentes au regard des programmes d'enseignement et les informations d'accueil peuvent être présentées par différents moyens techniques. L'arrivée de la génération $\mathrm{Y}$ est une opportunité pour améliorer l'informatisation du recrutement (et du système de santé.... ${ }^{[60]}$. Les étudiants apprécient de rencontrer des résidents de leur génération pour être éclairés et être à l'aise de poser toutes les questions concernant les programmes de formation ${ }^{[3]}$.

Selon des études effectuées dans le monde des affaires sur la génération $\mathrm{Y}$ comme ressource humaine, il est souvent utile de convier également les parents à des visites des milieux et partager avec eux l'information concernant les programmes ${ }^{[3]}$.

Un résumé des particularités et défis propres à la génération $Y$ avec les stratégies pédagogiques qui en découlent est présentée dans le tableau I. 


\section{Discussion}

\section{Discussion générale}

Chaque particularité de la génération $\mathrm{Y}$, telle que présentée dans les résultats issus de la littérature est reprise et discutée en fonction de l'expérience de l'auteure.

\section{L'enfant-roi devenu adulte}

Avec cette caractéristique générationnelle de l'enfant peu confronté à l'échec, les évaluations formatives et sommatives sont devenues plus complexes et demandent d'être particulièrement soignées. Cette caractéristique peut expliquer la difficulté de certains étudiants à se mettre en position d'ouverture par rapport à l'apprentissage. Il faut expliquer aux étudiants ce qu'est une saine position d'apprentissage car bien comprendre est un impératif pour cette génération. Il faut que toutes les attentes soient bien connues des étudiants avant chaque situation d'apprentissage et révisées périodiquement. Les conséquences de tout comportement inapproprié doivent également être discutées à l'avance.

\section{Victimes de surinvestissement}

Les nouveaux étudiants ont besoin de rétroaction fréquente sur leur performance, l'absence de commentaires ne signifiant plus une marque d'une bonne appréciation du travail effectué. Il y a place à l'amélioration de cet aspect de l'accompagnement des étudiants. Cette caractéristique confirme aussi l'importance de la proximité entre l'enseignant et l'apprenant.

\section{Activités d'équipe programmées à la minute}

Les étudiants de la génération Y sont des experts du réseautage, ce qui est très positif à l'ère de la médecine collaborative et l'auteure croit que les générations antérieures ont beaucoup à apprendre d'eux.

L'auteure a constaté dans la littérature que c'est d'abord la compétence et non l'ancienneté que les étudiants reconnaissent et valorisent et qui inspire leur confiance. Ils ont une conception différente de la hiérarchie et c'est pourquoi ils se sentent facilement les égaux des plus expérimentés.

Du fait de la surprotection qui leur a été attribuée et de la surestimation possible de leur capacité, les membres de la génération $Y$ peuvent osciller entre les deux pôles extrêmes de manque de confiance et d'excès de confiance («overconfidence »).

Il est nécessaire de détecter cet excès de confiance qui peut les mettre dans des situations d'apprentissage sans avoir les connaissances ou les habiletés prérequises. Pour le professeur, le fait de respecter et d'accueillir leurs lacunes aiderait l'étudiant à se mettre en position favorable à l'apprentissage. Il est préférable d'admettre leurs interrogations auprès des patients également.

\section{La peur au rendez-vous}

Au-delà de la formation classique, l'auteure croit que nous devons encourager la génération Y à s'investir dans un projet communautaire. Ils peuvent effectuer des interventions dans des milieux pour élèves en difficultés ou auprès des populations vulnérables de leurs environnements immédiats, par exemple. Ces projets permettent aussi de créer un esprit d'équipe parmi les étudiants, ce qui est important. Elle croit aussi qu'il faut valoriser les qualités de leadership et encourager les étudiants à donner l'exemple et à prendre des responsabilités, au-delà d'une formation théorique.

\section{Ni adolescent, ni adulte}

Au moment de leurs études, plusieurs étudiants ne sont pas encore autosuffisants tel que le rapporte la littérature ${ }^{[50]}$.

Selon l'auteure, la connaissance de ce stade de développement est primordiale et aide surtout à comprendre qu'ils ne peuvent être identiques à la génération qui les précède. Ils arrivent à maturité plus tard et ils vont continuer d' acquérir de la maturité parfois longtemps après avoir terminé leur résidence. Nous devons parfois les accompagner dans l'organisation, la gestion du temps et la prise de décision pour une plus longue période qu'auparavant. 
Le sens des responsabilités n'est pas inné ; son acquisition n'est pas spontanée et doit être un sujet de discussion et de sensibilisation auprès des étudiants.

\section{Équilibre travail/vie personnelle}

Même s'ils valorisent l'équilibre entre travail et vie personnelle, l'engagement envers la population de la part des futurs praticiens est essentiel. C'est un défi énorme. L'auteure croit qu'il faut faire confiance aux jeunes de la génération $Y$ car l'altruisme est un processus dynamique et longitudinal qu'il faut encourager avec persistance.

L'auteure croit que même s'ils sont en formation, nous avons beaucoup d'efforts à faire pour leur procurer des horaires académiques acceptables et flexibles.

\section{Nés avec une souris à la main}

L'auteure croit que le modèle de rôle est plus important que jamais. Comme ils trouvent l'information facilement (les savoirs sont devenus très disponibles), nous devons axer notre enseignement davantage sur le savoir-faire et le savoir-être d'autant qu'avec les technologies, ils ont moins bien appris à communiquer en personne. En observant les interactions interpersonnelles au sein de la population médicale et en les encourageant à parler d'eux, ils apprendront à mieux communiquer avec les autres.

Nous devons être une source d'inspiration pour les étudiants, en leur donnant la chance de découvrir l'excitation d'apprendre et ce tout au long de la vie. Il est aussi de notre rôle de les inciter à être rigoureux et critiques relativement à toutes les sources d'information qui doivent être probantes et fiables.

\section{Efficaces en activité multitôche ?}

Il sera plutôt difficile de déconstruire une telle attitude car le comportement semble très ancré. Néanmoins, l'auteure croit qu'ils doivent savoir que l'apprentissage en multitâche n'est pas efficace, surtout lorsqu'ils ont des difficultés d'apprentissage. L'effet non-verbal de désintérêt potentiel produit au quotidien par le multitâche est aussi non négligeable. Il faut donc définir les règles de ce qui est acceptable en classe et en stage et veiller à les respecter.

\section{Capacité d'apprentissage différente}

L'auteure considère que cet aspect générationnel est particulièrement préoccupant car, même s'ils excellent pour rechercher et obtenir de l'information, plusieurs n'ont pas les habiletés critiques et analytiques pour la comprendre et l'évaluer et manquent de flexibilité pour gérer les situations ambiguës, alors que ce sont des atouts très importants pour étudier et pratiquer la médecine. Ils sont à risque de difficultés scolaires plus précoces et plus importantes, ce qu'elle remarque chez certains étudiants.

Le soutien pédagogique avec plans individualisés tenant compte de leurs capacités est possible avec l'aide de conseillers pédagogiques et il faut les faire intervenir précocement durant le processus d'apprentissage, sinon l'escalade de sentiments d'incompétence s'installe et les place à risque d'échec. La rétroaction objective et constructive doit les aider à évaluer leurs forces et leurs faiblesses.

Dans l'ordre d'importance, l'évaluation de l'apprentissage ne doit pas prendre la place de l'apprentissage. Les évaluations ont pris une importance démesurée ils veulent une bonne note parfois pour le simple fait d'avoir assisté au cours. Il faut aider les apprenants à mettre l'emphase sur les compétences.

\section{Cours magistraux désuets}

Bien que les professeurs et même les autorités reconnaissent que les cours magistraux sont désuets, l'auteure considère qu'ils sont encore trop employés de façon classique parfois même en visioconférence sur plusieurs sites ce qui ne stimule pas l'apprentissage des étudiants de la génération Y. Une bonne présentation magistrale a parfois encore sa place mais il faut à tout le moins planifier leur participation active à des moments clés de la présentation, idéalement toutes les 15 minutes.

\section{Les dettes}

La littérature populaire parle beaucoup de ce phénomène très répandu chez les jeunes et les étudiants en médecine n'y échappent pas. L'auteure, dans son rôle 
de soutien aux étudiants, a constaté que les dettes peuvent forcer certains étudiants en médecine à continuer à tout prix malgré un certain désintérêt ou à faire un choix de résidence en fonction de la rémunération ou de la durée plutôt que du réel intérêt. Il est d'un intérêt de planifier des cours sur la gestion des finances ainsi que des ateliers sur l'orientation professionnelle tôt dans la formation médicale.

\section{Image sociétale réaliste}

Les curriculum vitae des étudiants candidats à la résidence sont le plus souvent impressionnants. Ils manifestent de grandes habiletés en musique ou en disciplines sportives de haut niveau. Ils sont ethnocentriques et voyagent beaucoup.

C'est un fait qu'ils peuvent paraître moins intéressés, moins impliqués, mais ce n'est pas nécessairement le cas, constate l'auteure. Leur identité ne se définit pas à partir de leur profession uniquement et ils ont besoin que nous leur expliquions la grande importance qu'ils ont pour leurs patients et la juste place que doivent occuper ces derniers dans leurs vies actives.

\section{Recrutement}

Une place spéciale pour les parents sur les sites Internet des programmes, une visite des milieux organisée à leur intention lors de la première journée, par exemple, pourraient être appréciése par les étudiants. Comme l'apprentissage de la médecine est une étape stressante et que les parents ont beaucoup d'importance pour cette génération, l'auteure comprend mieux pourquoi les étudiants la consultent parfois avec un parent et se demande si inviter les parents d'emblée lors des moments difficiles pourrait être aidant. Il pourrait s' agir notamment d'un moment profitable pour partager des conseils sur l'acquisition de l'autonomie de leur jeune.

Limites de l'étude

La robustesse des données présentées dans cet article réside dans le fait qu'elles résultent d'une revue exhaustive de la littérature, qui a permis de relever de nombreuses caractéristiques pertinentes de la génération Y.

Par contre, la synthèse de cette littérature s'est adossée sur une mise en perspective globale des différentes données, sans qu'une critique systématique de chacun des articles soit effectuée, à l'aide d'une grille d'analyse spécifique par exemple.

Étant donné que les caractéristiques des étudiants en médecine ont été enrichies par des caractéristiques des étudiants dans d'autres domaines en santé parce qu'ils partagent de nombreux points en commun, il peut tout de même y avoir une limite à l'interprétation des résultats. Cependant, malgré la variété des sources, comme la littérature est consensuelle et qu'il ne semble pas y avoir de contradiction quant aux caractéristiques et défis de la génération $\mathrm{Y}$ dans différents domaines qui rejoignent ceux de l'abondante littérature populaire, le biais apparaît mineur.

La revue de littérature a relevé des stratégies d'étude plutôt générales qui les rendent moins opérationnelles que ce qui était attendu, mais peuvent à tout le moins servir de guide et stimuler la réflexion et la discussion. Quoique la majorité des articles proviennent d'Amérique du Nord, en s'inspirant de la littérature populaire, tout porte à croire que la problématique de la génération Y peut être extrapolée à d'autres parties du monde. La nécessité de s'approprier des stratégies pédagogiques spécifiques à cette génération peut également s'étendre à d'autres populations, si l'auteure se fie sur son expérience professionnelle, la littérature populaire et les discussions informelles qu'elle a eues avec des professeurs d'ici et d'autres pays.

\section{Conclusion}

Cette synthèse de la littérature sur la génération Y permet de mettre en lumière plusieurs caractéristiques chez ces étudiants, constituant autant de défis pédagogiques à relever. Elle fournit des réponses à certains questionnements quant au rôle actuel de professeur de médecine et propose des pistes de stratégies pour mieux l'outiller. Elle répond aux questions de l'auteure et aux préoccupations ciblées dans le questionnaire exploratoire destiné aux professeurs. 
L'auteure retient plusieurs notions utiles dans son travail quotidien auprès des étudiants de tous les niveaux, parmi lesquelles : le soin à apporter lors de la remise des évaluations difficiles et la nécessité de favoriser la position d'apprentissage, le besoin de rétroaction fréquente chez un apprenant qui accepte mal la critique et gère mal les difficultés et les déceptions, la notion d'adulte émergent, l'omniprésence des technologies et son impact, l'expertise des étudiants dans le travail collaboratif, leur difficulté d'apprentissage, le poids qu'ils accordent à la qualité de vie qui est centrale et l'altruisme à développer. D'une façon générale, ces étudiants ne sont pas contestataires, ils veulent simplement comprendre.

Notre connaissance des caractéristiques spécifiques de la génération Y peut encore être enrichie et précisée. Les orientations à privilégier quant aux stratégies pédagogiques à mettre en œuvre doivent encore largement être documentées et validées. L'auteure a commencé à explorer des hypothèses de stratégies et à les évaluer en présentant sous forme de conférence interactive les résultats de sa synthèse de la littérature à des professeurs de différents milieux. Elle profite de ces occasions pour discuter plus en profondeur des façons de s'approprier et de rendre plus opérationnelles les stratégies proposées par la littérature.

Les jeunes de la génération Y sont de nouveaux bâtisseurs à qui on doit faire confiance. Plusieurs chercheurs croient que l'impact positif des générations $Y$ sera beaucoup plus grand que celui de toutes les générations antérieures ${ }^{[1]}$. Même s'ils ont un style direct et franc qui peut parfois choquer ${ }^{[42,69]}$, mieux vaut comprendre leurs perspectives et réaliser qu' elles sont le reflet de la culture contemporaine : cette génération fait exactement ce que leur ont demandé leurs parents, leurs professeurs et les médias ${ }^{[6]}$. Mieux les connaître contribue à améliorer notre efficacité et notre créativité de professeurs et nous permet probablement de revoir et d'évaluer nos propres valeurs et de profiter de leur expertise. Après tout, ne sont-ils pas les opérateurs de changement que toutes les générations aimeraient constater?

\section{Remerciements}

L'auteure tient à remercier les collègues suivants pour la révision du manuscrit et les conseils : Norma Bélanger de l'Université Laval, Richard Boulé, Martin Fortin, Tania Fayad, de l'Université de Sherbrooke et Susie Bernier, Tarek Bouhali, José Almirall du Centre de santé et de services sociaux de Chicoutimi.

\section{Références}

1. Burkus D. Leading the next generation: six ways to turn millennials into followers. LeaderLab 2010;1:18-21.

2. Meister JC, Willyerd K. Mentoring millennials. Harv Bus Rev 2010;88:68-72.

3. Schlitzkus LL, Schenarts KD, Schenarts PJ. Is your residency program ready for Generation Y? J Surg Educ 2010;67:108-11.

4. Kurup V. The new learners - Millennials!! Int Anesthesiol Clin 2010;48:13-25.

5. Moreno-Walton L, Brunett P, Akhtar S, DeBlieux PM. Teaching across the generation gap: a consensus from the Council of Emergency Medicine Residency Directors 2009 academic assembly. Acad Emerg Med 2009;16 Suppl 2:S19-24.

6. Twenge JM. Generational changes and their impact in the classroom: teaching Generation Me. Med Educ 2009;43:398-405.

7. Howell L, Joad J, Callahan E, Servis G, Bonham A. Generational forecasting in academic medicine: a unique method of planning for success in the next two decades. Acad Med 2009;84:985-93.

8. Lavoie-Tremblay M, Leclerc E, Marchionni C, Drevniok $\mathrm{U}$. The needs and expectations of generation $\mathrm{Y}$ nurses in the workplace. J Nurses Staff Dev 2010;26:2-8; quiz 9-10.

9. Johnson SA, Romanello ML. Generational diversity: teaching and learning approaches. Nurse Educ 2005;30:212-6.

10. Mangold K. Educating a new generation: teaching baby boomer faculty about millennial students. Nurse Educ 2007;32:21-3.

11. Chambers DW. Generations. J Am Coll Dent 2005;72:27-35. 
12. Shangraw RE, Whitten CW. Managing intergenerational differences in academic anesthesiology. Curr Opin Anaesthesiol 2007;20:558-63.

13. Lower J. Brace yourself. Here comes generation Y. Crit Care Nurse 2008;28:80-5; discussion 3-4.

14. Venne VL, Coleman D. Training the Millennial learner through experiential evolutionary scaffolding: implications for clinical supervision in graduate education programs. J Genet Couns 2010;19:554-69.

15. Chambers PD. Tap the unique strengths of the millennial generation. Nurs Manage 2010;41:36-9.

16. Jamieson I. Generation $Y$ - why nursing must retain this workforce. Nurs N Z 2009;15:18-20.

17. Lyons ST, Duxbury L, Higgins C. An empirical assessment of generational differences in basic human values. Psychol Rep 2007;101:339-52.

18. Myers KK, Sadaghiani K. Millennials in the Workplace: A Communication Perspective on Millennials' Organizational Relationships and Performance. J Bus Psychol 2010;25:225-38.

19. Borges NJ, Manuel RS, Elam CL, Jones BJ. Differences in motives between Millennial and Generation X medical students. Med Educ 2010;44:570-6.

20. Eley D. Why the fuss about Generation Y? Med Teach 2010;32:348-9.

21. Jones R. Generation $\mathrm{Me}$ and us. Med Educ 2009;43:819.

22. Wilson I, Harding D. I will only work from 2.00 to 5.00.... Med Educ 2009;43:394-5.

23. Mitchell DA. Generation Z - striking the balance: healthy doctors for a healthy community. Aust Fam Physician 2008;37:665-7.

24. Stockinger ZT, Ellis MS, McSwain NE, Jr. Residents and medical students in the 21st century: better, worse, or just different? Bull Am Coll Surg 2004;89:21-3.

25. Ramsay M. The new generation of graduating anesthesia residents: what is the impact on a major tertiary referral private practice medical center? Curr Opin Anaesthesiol 2007;20:568-71.

26. Kapur PA. The impact of new-generation physicians on the function of academic anesthesiology departments. Curr Opin Anaesthesiol 2007;20:564-7.

27. Perler BA. Presidential Address. When I grow up, I want to be successful like daddy: I just don't want to be a doctor. J Vasc Surg 2007;45:627-34.
28. Garcia C, Davison M. Awareness of generational differences is the first step. Arch Dermatol 2007;143:120-1.

29. Skinner CA. Re-inventing medical work and training: a view from generation X. Med J Aust 2006;185:356.

30. Johnston S. See one, do one, teach one: developing professionalism across the generations. Clin Orthop Relat Res 2006;449:186-92.

31. Borges NJ, Manuel RS, Elam CL, Jones BJ. Comparing millennial and generation $\mathrm{X}$ medical students at one medical school. Acad Med 2006;81:571-6.

32. Shah SI. A 1920 doctor in the Year 2000. Patient Educ Couns 2006;60:100-1.

33. Waddell JP. Generations of training. Can J Surg 2004;47:85-6.

34. Larson DL. Bridging the generation X gap in plastic surgery training: part 1 . Identifying the problem. Plast Reconstr Surg 2003;112:1656-61.

35. Craven JE, 4th. The generation gap in modern surgery: a new era in general surgery. Arch Surg 2002;137:257-8.

36. Meyer AA, Weiner TM. The generation gap: perspectives of a program director. Arch Surg 2002;137:26870.

37. Organ $\mathrm{CH}$, Jr. The generation gap in modern surgery. Arch Surg 2002;137:250-2.

38. Gorrindo T, Groves JE. Web searching for information about physicians. JAMA 2008;300:213-5.

39. Williamson T. The generation $Y$ adult learning theory. An analysis of the new generation of radiography students. 2009 [On-line] Disponible sur : http:// www.rt-image.com/042709GenerationY.

40. Musson DM. Personnality and medical education. Med Educ 2009;43:395-7.

41. Berthold J. Is the generation gap a growth opportunity? ACP Internist 2008. [On-line] Disponible sur : http://www.acpinternist.org/archives/2008/04/ two.htm

42. Howells L, Servis G, Bonham A. Multigenerational challenges in academic medicine: UCDavis's responses. Acad Med 2005;80:527-32.

43. Berenson AB. Presidential address: from generation to generation. Am J Obstet Gynecol 2005;192:1783-7.

44. Earle V, Myrick F, Yonge O. Preceptorship in the intergenerational context: an integrative review of the literature. Nurse Educ Today 2011;31:82-7. 
45. Keepnews DM, Brewer CS, Kovner CT, Shin JH. Generational differences among newly licensed registered nurses. Nurs Outlook 2010;58:155-63.

46. Zimmerman B. Mentoring the millennials. NASN Sch Nurse 2010;25:12.

47. Cubit KA, Ryan B. Tailoring a graduate nurse program to meet the needs of our next generation nurses. Nurse Educ Today 2011;31:65-71.

48. Smith S, Malone J, Agwu C, Clark AP. Millennials and their value cohort: how to educate them. Clin Nurse Spec 2009;23:289-92.

49. Gibson SE. Intergenerational communication in the classroom: recommendations for successful teacherstudent relationships. Nurs Educ Perspect 2009;30:37-9.

50. Pardue KT, Morgan P. Millennials considered: A new generation, new approaches, and implications for nursing education. Nurs Educ Perspect 2008;29:74-9.

51. Swenson C. Next generation workforce. Nurs Econ 2008;26:64-5.

52. Arhin AO, Cormier E. Using deconstruction to educate Generation Y nursing students. J Nurs Educ 2007;46:562-7.

53. Widger K, Pye C, Cranley L, Wilson-Keates B, Squires $M$, Tourangeau A. Generational differences in acute care nurses. Nurs Leadersh (Tor Ont) 2007;20:49-61.

54. Skiba DJ, Barton AJ. Adapting your teaching to accommodate the net generation of learners. (Online) J Issues Nurs 2006;11:5.

55. Walker JT, Martin T, White J, et al. Generational (age) differences in nursing students' preferences for teaching methods. J Nurs Educ 2006;45:371-4.

56. Hu J, Herrick C, Hodgin KA. Managing the multigenerational nursing team. Health Care Manag (Frederick) 2004;23:334-40.

57. Arhin AO, Johnson-Mallard V. Encouraging alternative forms of self expression in the generation $\mathrm{Y}$ student: a strategy for effective learning in the classroom. ABNF J 2003;14:121-2.
58. Parsons LC. Converging values: matures, boomers, xers, and nexters in the health care workforce. SCI Nurs 2002;19:25-7.

59. Shreeve MW. The Digital Bridge: A Boomer's View of Course Management Software in the Gen Y Classroom. J Chiropr Educ 2009;23:174-7.

60. Yee KC, Miils E, Airey C. Perfect match? Generation $\mathrm{Y}$ as change agents for information communication technology implementation in healthcare. Stud Health Technol Inform 2008;136:496-501.

61. Piper LE. The Generation-Y workforce in health care: the new challenge for leadership. Health Care Manag (Frederick) 2008;27:98-103.

62. Mills E, Airey C, Yee KC. Generation Y in healthcare: the need for new socio-technical consideration for future technology design in healthcare. Stud Health Technol Inform 2007;130:169-79.

63. Yee KC. Bermuda Triangle or three to tango: generation Y, e-health and knowledge management. Stud Health Technol Inform 2007;129:1184-8.

64. Hanley KJ. Overcoming the generational gap. N Y State Dent J 2005;71:6.

65. Hewlett SA, Sherbin L, Sumberg K. How Gen Y and Boomers will reshape your agenda. Harv Bus Rev 2009;87:71-6, 153.

66. Ludwig G. Generation Y why you can't connect. JEMS 2009;34:30.

67. Black A. GenY : Who are they and how they learn. Educ Horiz 2010;88:92-101.

68. Webster LA. Sharpen your communication skills. Here comes generation Y. MGMA Connex 2005;5:42-7.

69. Simard S. Génération Y Attirer, motiver et conserver les jeunes talents. Montréal : Les éditions Viséo; 2007.

Correspondance et offprints: Francine Boulé 305 St-Vallier, Chicoutimi, QC Canada G7G 4W4

Mailto : Francine.boule@usherbrooke.ca 